\title{
Cluster Distances using Delta Scuti Stars; The Open Clusters Praesepe and NGC 7062
}

\author{
Torben Arentoft \\ University of Brussels (VUB), Pleinlaan 2, B-1050 Brussels, Belgium
}

Lars M. Freyhammer

Royal Observatory of Belgium, Ringlaan 3, B-1180 Brussels, Belgium

University of Brussels (VUB), Pleinlaan 2, B-1050 Brussels, Belgium

\begin{abstract}
Asteroseismology of multiperiodic $\delta$ Scuti stars is a powerful tool for providing estimates of distances to the stars being analyzed. Especially $\delta$ Scuti stars in clusters offer good possibilities for determining asteroseismic distances, as the stellar models are constrained by assuming common values for chemical composition, age and distance. Recently, we detected a large population of $\delta$ Scuti stars in the open cluster NGC 7062. On the basis of these results, we discuss the potential of extending the asteroseismic distance scale for $\delta$ Scuti stars to include extra-galactic stellar clusters. Especially high-amplitude $\delta$ Scuti stars, pulsating in at least 2 radial modes, offer promising possibilities for doing this.
\end{abstract}

\section{Introduction}

High-amplitude $\delta$ Scuti stars (HADS) are promising galactic and inter-galactic distance indicators. This is due to the fact that period-luminosity (or even $P L C Z$ ) relations can be derived for these stars. The use of HADS as distance indicators is discussed by Freyhammer \& Petersen (these proceedings).

Here we discuss the use of multiperiodic $\delta$ Scuti stars in determining distances to other galaxies. The main goal of observing (galactic) $\delta$ Scuti stars is to apply them in asteroseismic analysis of the interior of the stars. This can be done by matching observed and theoretical frequency ratios of radial modes of different radial order $n$, and thereby finetune the model parameters. An estimate of the distance to the star is also derived in this process. Especially interesting are the high-amplitude double-mode $\delta$ Scuti stars, which pulsates in the fundamental mode and the first overtone. A notorius problem in $\delta$ Scuti star research is the difficulty of mode-identification. This can be obtained through frequency ratio fitting, but the procedure is dangerous if too many frequencies are excited simultaneously - a frequency ratio expected for two radial modes may turn up between two non-radial modes, as discussed by Poretti (2000). For this reason are the double-mode HADS the most promising objects: for those stars is the mode-identification provided. 


\section{The used method}

The method we use, was used to obtain density, and a distance in excellent agreement with the HIPPARCOS distance, for FG Vir by Viskum et al. (1998). We use a scaled $2.2 \mathrm{M}_{\odot} \delta$ Scuti model calculated by Christensen-Dalsgaard (1993). The following relation for radial modes, similar to a normal PLC-relation, is found:

$$
f(n)=a_{n}\left(\frac{L}{L_{\odot}}\right)^{b_{n}}\left(\frac{T_{e f f}}{5777 K}\right)^{3.07}
$$

Details and values for $a_{n}$ and $b_{n}$ can be found in Arentoft et al. (1998). Frequency ratios are used as they are much less sensitive to stellar parameters than the absolute frequency values. For example, Kjeldsen et al. (1998) showed that the ratios are virtually unaffected by $v_{\text {rot }}$ of the star, something which is not true, even for the radial frequencies themselves. Initial estimates of luminosity and $T_{\text {eff }}$ derived from photometry are used to limit the possible radial orders. If mode identification has been achieved by other means, (like in FG Vir, Viskum et al. 1998), Eq. 1 can be used to calculate model ratios for the observed radial modes. Otherwise the mode identification can, if the frequency spectrum of the star is relatively simple, be achieved by matching observed and calculated frequency ratios. When the radial modes have been located and identified, the ratios can be used to adjust the basic parameters of the model. This leads to refined values of effective temperature, density, mass, radius - and distance. The method was also used by Arentoft et al. (1998), who found a mean asteroseismic distance modulus for three Praesepe stars of $6.27 \pm 0.03 \mathrm{mag}$, in agreement with the HIPPARCOS distance modulus.

\section{NGC 7062}

Using a 2.6-m telescope, we observed this more distant, but still Galactic, open cluster. We detected at least $13 \delta$ Scuti stars within $6^{\prime}$ by $6^{\prime}$ field (Freyhammer et al. 2001). The distance modulus, determined from the colour-magnitude diagram is $12.8 \pm 0.4 \mathrm{mag}$. Differential photometry to mmag precision was obtained. For one of the stars, NGC7062 V1, 9 frequencies were detected with amplitudes below 4 mmag. Using the method described here, we could reconcile two of them with radial modes - however, the precision of the individual frequencies (from 4 nights of data) were not sufficiently high to improve the distance modulus. But the obtained precision in the photometry serves as a base for a discussion on what is needed to use the $\delta$ Scuti stars as distance indicators for galaxies.

\section{Discussion}

The use of the method described in Sect. 2 can be extented to other galaxies by utilising larger telescopes. With a telescope in the $4 \mathrm{~m}$ class, mmag photometry can be obtained in the Magellanic Clouds (the distance modulus for LMC is $\sim 18.5 \mathrm{mag}$ ). For more distant stars, for example in the Carina galaxy at a distance modulus of about 20, a star similar NGC 7062 V1 would require an 8-m 
telescope to provide sufficiently high precision. Mateo et al. (1998) discovered 20 HADS in Carina using a $4.0 \mathrm{~m}$ telescope. The precision in their photometry was about $0.05-0.1 \mathrm{mag}$, and they found the variables to follow a $P L$ relation. This precision can obviously be improved with larger telescopes, but their results show that for such distant objects, one should focus on high-amplitude doublemode $\delta$ Scuti stars. For stars e.g. in Carina, the limiting factor may not be the precision of the obtained light curves, but rather the difficulty of deriving reasonable basic stellar parameters for the oberved stars in order to constrain the parameters of the models. This is again an argument for focussing on highamplitude double-mode $\delta$ Scuti stars, as the mode-identification is much easier in these stars. The observations should cover a long time base, at least of the order of months (not necessarily continously), in order to assure high frequency resolution.

Acknowledgments. TA acknowledges financial support from the Belgian Fund for Scientific Research (FWO). This project was supported by the Flemish Ministry for Foreign Policy, European Affairs, Science and Technology, under contract BIL 99/2. LMF acknowledges support from IUAP P4/05 financed by the Belgian DWTC/SSTC.

\section{References}

Arentoft, T., Kjeldsen, H., Nuspl, J. et al., 1998, A\&A, 338, 909

Christensen-Dalsgaard, J., 1993, in Inside the stars, ASP Conf. Ser. Vol. 40, eds. W.W. Weiss and A. Baglin (San Francisco: ASP), 483

Freyhammer, L.M., Arentoft, T., \& Sterken, C., 2001, A\&A, 369, 561

Kjeldsen, H., Arentoft, T., Bedding, T.R. et al., 1998, in Structure and Dynamics of the Interior of the Sun and Sun-like Stars, SOHO 6/GONG 98, 385

Mateo, M., Hurley-Keller, D., \& Nemec, J., 1998, AJ, 115, 1856

Poretti, E., 2000, in Delta Scuti and related stars, ASP Conf. Ser. Vol. 210, eds. M. Breger and M. Montgomery (San Francisco: ASP), 45

Viskum, M., Kjeldsen, H., Bedding, T.R. et al., 1998, A\&A 335, 549 